\title{
HIDRÓLISE DO ÓLEO DE Azadirachta indica EM ÁGUA SUBCRÍTICA E DETERMINAÇÃO DA COMPOSIÇÃO DOS TRIACILGLICERÍDEOS E ÁCIDOS GRAXOS POR CROMATOGRAFIA GASOSA DE ALTA RESOLUÇÃO A ALTA TEMPERATURA E CROMATOGRAFIA GASOSA DE ALTA RESOLUÇÃO ACOPLADA À ESPECTROMETRIA DE MASSAS
}

\author{
Jair Sebastião da Silva Pinto*\# e Fernando Mauro Lanças \\ Instituto de Química de São Carlos, Universidade de São Paulo, 13565-900 São Carlos - SP, Brasil
}

Recebido em 20/5/09; aceito em 28/7/09; publicado na web em 8/1/10

\begin{abstract}
HYDROLYSIS OF Azadirachta indica OIL USING SUBCRITICAL WATER AND DETERMINATION OF TRIACYLGLYCERIDES AND FAT ACIDS BY HT-HRGC-FID AND HRGC-MS. The development of modern analytical tools plays an important role in quality control. The main purpose of this study was to explore the use of subcritical water as a versatile analytical tool, employed simultaneously as a reagent and solvent, as well as the application of high temperature-high resolution gas chromatography (HT-HRGC) to develop a procedure for the analysis of triacylglycerides and fatty acids in Azadirachta indica A. Juss. (Neem) oil without the need for solvents, chemical reagents, or catalytic agents. The developed method presented satisfactory results and is in agreement with the concepts of Green Analytical Chemistry (GAC).
\end{abstract}

Keywords: Neem oil; subcritical water; Green Analytical Chemistry (GAC).

\section{INTRODUÇÃO}

Azadirachta indica A. Juss mais conhecida como Nim, originária de Burma ou Mianmar, na Ásia, é encontrada na Índia e regiões tropicais e subtropicais do mundo, como Austrália, África, Ilhas do Pacífico e Américas. Pertence à família Meliaceae, como a santa-bárbara ou cinamomo, cedro e mogno. A literatura descreve-a como uma planta medicinal usada há séculos na Índia, com propriedades bactericidas, fungicidas, nematicidas, anti-inflamatória ${ }_{2}$ anti-hiperglicêmica, repelente de insetos, entre outras. ${ }^{1}$ Estas propriedades se devem aos diversos terpenoides distribuídos na planta (semente, folha, casca). ${ }^{2-4}$

No Brasil a planta foi introduzida inicialmente em 1986 pelo Instituto Agronômico do Paraná (IAPAR), com sementes originárias das Filipinas. Na década passada, com a divulgação de suas propriedades, o cultivo comercial de Nim se disseminou por vários estados do Brasil. Como os terpenoides se distribuem pela planta, todas as partes são passíveis de uso, desta maneira utiliza-se o óleo extraído das sementes, a torta resultante, folhas, frutos e casca. O óleo da semente de Nim (A. indica) é muito amargo e contém vitamina E e aminoácidos essenciais. Dados de literatura indicam que o óleo é constituído pelos ácidos graxos oleico, palmítico, esteárico, linoleico, mirístico, linolênico e araquidônico. ${ }^{5} \mathrm{O}$ grande potencial para exploração agrícola, medicinal e industrial justifica o desenvolvimento de metodologias analíticas modernas visando o controle de qualidade e de fraudes. Uma possível rota de adulteração do óleo de Nim seria a mistura com outros óleos vegetais de baixo valor comercial. Uma das maneiras de detectar a adulteração é monitorar a composição dos triacilglicerídeos (TAG) e ácidos graxos (AG). ${ }^{6,7}$ A cromatografia gasosa de alta resolução a alta temperatura (HTHRGC) é uma técnica adequada para análise dos TAG. ${ }^{8-10}$

Hidrólise de TAG a partir de óleos e gorduras para obter glicerol e ácidos graxos (AG) é uma importante reação da indústria oleoquímica. ${ }^{11}$ Essa reação é homogênea de primeira ordem e se desenvolve na fase oleosa. ${ }^{12}$ Segundo vários autores, a hidrólise ocorre em etapas

*e-mail: jsspinto@esalq.usp.br

\# Endereço atual: Escola Superior de Agricultura Luiz de Queiroz, Universidade de São Paulo, 13418-900 Piracicaba - SP, Brasil. e é reversível. ${ }^{10-12}$ Os triacilglicerídeos (TAG) são hidrolisados a diacilglicerídeos (DAG), monoacilglicerídeos (MAG) e glicerol. Para cada etapa há liberação de um ácido graxo.

O método tradicional de análise dos TAG consiste de diversas etapas. ${ }^{13}$ Em resumo, os TAG são convertidos a AG e glicerol. Os AG são então esterificados aos seus respectivos metil ésteres, os quais são identificados e quantificados por cromatografia gasosa. Neste método são utilizados solventes orgânicos, catalisadores ácidos e/ ou básicos e reagentes.

A hidrólise de TAG aos seus respectivos ácidos graxos também pode ser realizada em água subcrítica. ${ }^{10,14}$ Isto possibilita o desenvolvimento de um procedimento analítico ambientalmente amigável, pois não se faz necessário o uso de solventes orgânicos, reagentes e catalisadores.

Água no estado subcrítico $\left(100^{\circ} \mathrm{C}<\mathrm{T}<374,2^{\circ} \mathrm{C}\right)$ e supercrítico $\left(\mathrm{T}=374,2^{\circ} \mathrm{C}, \mathrm{P}=22,05 \mathrm{MPa}\right)$ tem despertado grande interesse como reagente e solvente ambientalmente amigável. Água subcrítica tem sido utilizada em estudos de hidrólise de biomassa, ${ }^{15}$ extração a partir do farelo de arroz de substâncias funcionais, ${ }^{16,17}$ proteínas e aminoácidos ${ }^{18}$ hidrólise de celulose microcristalina, ${ }^{19}$ estudos da cinética da hidrólise de biopolímeros, ${ }^{20}$ entre outros que se mostram muito promissores. Com o aumento da temperatura, até $250^{\circ} \mathrm{C}$, e sob pressão, suficiente para manter o estado líquido, ocorre o aumento do produto iônico da água $\left(\mathrm{K}_{\mathrm{w}} \sim 10^{-11}\right)$, o qual favorece o desenvolvimento de reações de mecanismos iônicos. ${ }^{18,21}$ Também se verifica a redução da polaridade, com o aumento da temperatura, a qual é devida à diminuição da constante dielétrica. ${ }^{16}$ Nessa região subcrítica a água possui propriedades (densidade e constante dielétrica) similares ao de solvente orgânico a temperatura ambiente. ${ }^{16,22}$ Desta maneira, a água subcrítica pode participar de várias reações químicas como solvente e reagente, sendo uma alternativa tecnicamente viável e ecologicamente aceitável ao uso dos solventes orgânicos, pois tem a vantagem de ser atóxica, não inflamável, não explosiva e de baixo custo.

O presente estudo teve por objetivo, portanto, explorar o uso da água subcrítica como uma ferramenta analítica versátil na hidrólise do óleo de Nim (A. indica), sem o uso de qualquer solvente ou catalisador, seguida da análise direta dos AG resultantes por cromatografia gasosa de alta resolução. Também foi objetivo do presente estudo o 
uso da cromatografia gasosa de alta resolução a alta temperatura com detector de ionização em chama (HT-HRGC-FID) para análise direta dos TAG do óleo de Nim (A. indica) sem qualquer etapa de preparação e contribuir para o desenvolvimento de procedimentos analíticos que se enquadrem no conceito de Química Analítica Verde. ${ }^{23,24}$

\section{PARTE EXPERIMENTAL}

\section{Reagentes e materiais}

A água foi destilada e, posteriormente, deionizada em sistema Milli Q Millipore (Bedford, MA, USA). Hexano grau PA foi fornecido pela Merck (Rio de Janeiro, RJ, Brasil), Metanol grau HPLC fornecido pela Mallinckrodt Baker Inc. (Paris, Kentucky, USA), e a mistura 61C de metil ésters de ácidos graxos fornecida pela PolyScience (Niles, IL, USA). Uma cela de hidrólise de aço inox foi confeccionada ( $7.5 \mathrm{~cm}$ x $0.4 \mathrm{~cm}$ i.d.), e um forno de aquecimento de um cromatógrafo da CG Instrumentos foi utilizado (São Paulo, SP, Brasil).

\section{Amostras}

Uma amostra de óleo de Nim (A. indica), não refinado, foi comprada no comércio local de Campinas (SP) e utilizada sem qualquer tratamento prévio.

\section{Hidrólise}

Foram utilizadas $150 \mathrm{mg}$, em triplicata, do óleo de Nim (A. indica) e $1000 \mathrm{mg}$ de água deonizada, previamente desaerada com hélio. A mistura colocada na cela de hidrólise foi fechada nas extremidades e colocada, na posição vertical, no forno da CG Instrumentos na temperatura de $280{ }^{\circ} \mathrm{C}$ por $40 \mathrm{~min}$. A pressão na cela de hidrólise foi estimada, baseada na pressão de vapor saturado, em aproximadamente 6,5 MPa.

Depois de efetuada a hidrólise, a mistura resultante, constituída de duas fases, foi separada. A fase aquosa foi descartada. A fase oleosa foi analisada inicialmente por cromatografia gasosa de alta resolução a alta temperatura com detector de ionização em chama (HT-HRGCFID), para confirmar a total conversão dos TAG em AG e glicerol.

\section{Triacilglicerídeos por HT-HRGC/FID}

O óleo comercial de Nim (A. indica) e a fração oleosa, resultante da hidrólise, foram diluídos, cada um, com $2 \mathrm{~mL}$ de hexano PA e injetados $(1 \mu \mathrm{L})$ no cromatógrafo a gás da Hewlett Packard modelo HP 5890 Série II com injetor split/splitless a $370{ }^{\circ} \mathrm{C}$ e o detector de ionização de chama (FID) a $380{ }^{\circ} \mathrm{C}$. A separação cromatográfica foi efetuada com uma coluna Croma-5 HT [5\% fenil, 95\% dimetilpolissiloxano entrecruzada e imobilizada] ( $9 \mathrm{~m}$ x $0,25 \mathrm{~mm}$ i.d x 0,09 $\mu \mathrm{m})$ com uma programação de temperatura iniciando em $150{ }^{\circ} \mathrm{C}$ por 1 min, e então de 150 a $380{ }^{\circ} \mathrm{C}$ a $8{ }^{\circ} \mathrm{C} / \mathrm{min}$ até $380^{\circ} \mathrm{C}$, mantendo-se nesta temperatura por $10 \mathrm{~min}$. O fluxo da coluna foi de $3,5 \mathrm{~mL} / \mathrm{min}$, com hidrogênio como gás de arraste, e split de 1:10. Os dados foram coletados em um integrador HP 3396A.

A identificação dos triacilglicerídeos foi efetuada comparandose com o tempo de retenção $\left(\mathrm{t}_{\mathrm{R}}\right)$ do padrão de trioleína-T54, tomado como padrão de referência de $t_{R}$.

\section{Ácidos graxos por HRGC/FID}

A identificação dos ácidos graxos, da fração oleosa da hidrólise, foi realizada utilizando-se cromatografia gasosa de alta resolução com detector de ionização em chama (HRGC-FID).
A separação cromatográfica foi efetuada utilizando-se uma coluna Croma-100 [Polietilenoglicol] (30 m x 0,25 mm i.d x 0,3 $\mu \mathrm{m}$ ) com a seguinte programação de temperatura: $100{ }^{\circ} \mathrm{C}$ por $2 \mathrm{~min}$, e então de 100 a $250{ }^{\circ} \mathrm{C}$ a $10{ }^{\circ} \mathrm{C} / \mathrm{min}$, e $250{ }^{\circ} \mathrm{C}$ por $10 \mathrm{~min}$. O fluxo de hidrogênio utilizado como gás de arraste foi de $1,2 \mathrm{~mL} / \mathrm{min}$. $\mathrm{O}$ injetor foi operado no modo split a $250{ }^{\circ} \mathrm{C}$ como uma razão de split de $1: 10$, e o detector a $300{ }^{\circ} \mathrm{C}$. A velocidade linear de trabalho foi de $39 \mathrm{~cm} / \mathrm{s}$. As identificações foram efetuadas comparando com os tempos de retenção das misturas padrão.

\section{Ésteres metílicos de ácidos graxos (FAME) por HRGC/MS}

Para verificar a possibilidade de alguma alteração, ou decomposição dos ácidos graxos devido a alta temperatura de hidrólise, foi realizada a análise de FAME do óleo, ${ }^{25}$ em duplicata, sem passar por qualquer processo prévio.

Nessa análise foram pesados $35 \mathrm{mg}$ do óleo de Nim (A. indica), em um tubo de vidro, com tampa, e foi adicionado $0,5 \mathrm{~mL}$ de solução metanólica de hidróxido de sódio 0,5 N. A seguir, o tubo foi fechado e o conteúdo aquecido em banho-maria a $90{ }^{\circ} \mathrm{C}$. Depois de $10 \mathrm{~min}$ o tubo foi retirado do banho-maria e resfriado em banho de gelo. Então, $1,5 \mathrm{~mL}$ de solução esterificante (cloreto de amônio, metanol e ácido sulfúrico) foi adicionada ao tubo, o mesmo foi fechado e aquecido novamente em banho-maria a $90{ }^{\circ} \mathrm{C}$ por $10 \mathrm{~min}$. $\mathrm{O}$ tubo foi resfriado, em banho de gelo, e nele adicionados $5 \mathrm{~mL}$ de hexano e $10 \mathrm{~mL}$ de água destilada. O tubo foi agitado e, após a separação das fases, a camada superior (usualmente $1 \mu \mathrm{L}$ ) foi analisada por cromatografia gasosa para identificação e quantificação dos FAMEs.

A identificação dos ésteres metílicos foi realizada utilizando-se cromatografia gasosa de alta resolução acoplada a um espectrômetro de massas da Shimadzu modelo GCMS-QP 5000 com injetor split/ splitless. A separação cromatográfica foi efetuada usando-se uma coluna Croma-5 [5\% fenil, 95\% dimetilpolissiloxano entrecruzada e imobilizada] ( $30 \mathrm{~m} \times 0,25 \mathrm{~mm}$ i.d $\times 0,3 \mu \mathrm{m}$ ) com a seguinte programação de temperatura: $150{ }^{\circ} \mathrm{C}$ por $5 \mathrm{~min}$, e então de 150 a 180 ${ }^{\circ} \mathrm{C}$ a $5^{\circ} \mathrm{C} / \mathrm{min}, 180{ }^{\circ} \mathrm{C}$ por $10 \mathrm{~min}, 180$ a $300{ }^{\circ} \mathrm{C}$ a $8{ }^{\circ} \mathrm{C} \%$ min. O split foi $1: 10$, a velocidade linear de $38 \mathrm{~cm} / \mathrm{s}$, o gás de arraste foi hélio ultrapuro, com temperatura do injetor de $250{ }^{\circ} \mathrm{C}$. As identificações foram efetuadas comparando com os tempos de retenção das misturas padrão e os espectros de massas no modo Scan.

Estimativa da composição triacilglicerídica do óleo de Nim (A. indica) a partir da composição de seus ácidos graxos

Tendo em vista a complexidade do cálculo da composição dos triacilglicerídeos empregou-se um software de análise que se baseia na composição dos ácidos graxos. ${ }^{25,26} \mathrm{O}$ software agrupa os triacilglicerídeos calculados pelo número de átomos de carbono com a notação $\mathrm{TN}$, sendo $\mathrm{T}$ para designar triacilglicerídeos e $\mathrm{N}$ o número de átomos de carbono. Então, T54 seria um triacilglicerídeo constituído por 3 ácidos graxos com 54 átomos de carbono.

Esses dados, por meio do software, foram comparados com os obtidos por HT-HRGC/FID.

\section{RESULTADOS E DISCUSSÃO}

\section{HT-HRGC-FID}

A HT-HRGC é uma técnica de grande utilidade em química analítica, mas curiosamente seu uso é muito pouco difundido. Apresenta a possibilidade de efetuar rapidamente análises de óleos e gorduras, sem os artifícios de esterificações que consomem vários reagentes e tempo. 
A identificação e quantificação dos triacilglicerídeos do óleo de $\operatorname{Nim}$ (A. indica) foram realizadas sem maiores dificuldades empregando-se HT-HRGC/FID.

A Figura 1 apresenta o perfil de triacilglicerídeos com picos bem característicos e a presença de ácidos graxos livres. Foi empregado o padrão de trioleina (T54) para comparar o tempo de retenção.

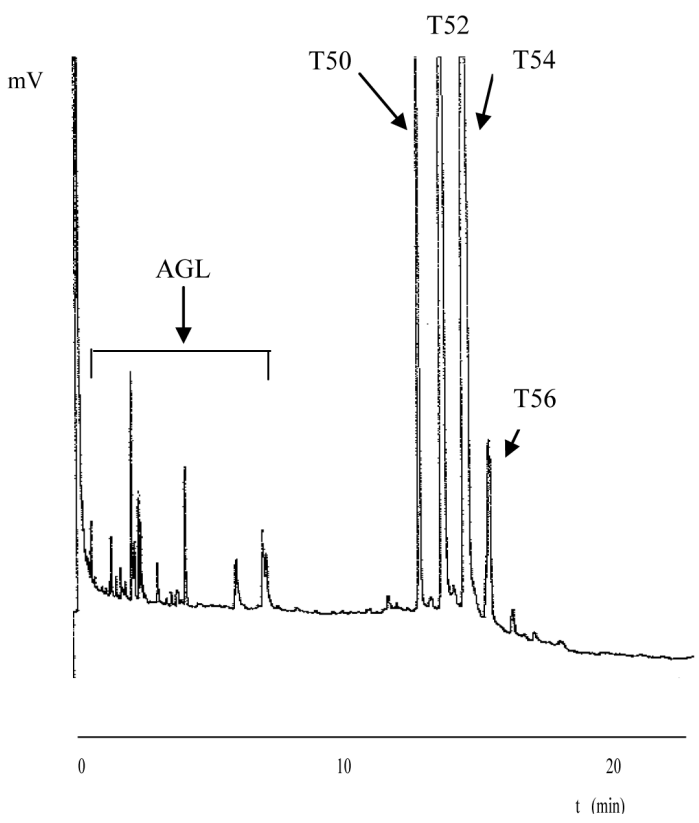

Figura 1. Cromatograma dos triacilglicerídeos do óleo de Nim (A. indica) por HT-HRGC/FID. AGL: Ácidos graxos livres

\section{FAME-HRGC/MS e HRGC-FID}

Na Tabela 1 tem-se a comparação da composição dos ácidos graxos determinados por FAME-HRGC/MS e HRGC/FID e, também, dados de literatura.

A composição de ácidos graxos (Tabela 1) foi equivalente ao descrito na literatura, ${ }^{5}$ com exceção dos ácidos araquidônico e mirístico não encontrados nos produtos de hidrólise.

Tabela 1. Composição de ácidos graxos do óleo de Nim (A. indica)

\begin{tabular}{|c|c|c|c|}
\hline Ácido Graxo & $\begin{array}{c}\text { FAME } \\
\text { HRGC-MS } \\
(\%)\end{array}$ & $\begin{array}{c}\text { Água Subcrítica } \\
\text { HRGC-FID } \\
(\%)\end{array}$ & $\begin{array}{c}\text { Literatura }^{5} \\
(\%)\end{array}$ \\
\hline $\begin{array}{l}\text { Ác. Mirístico } \\
\text { C 14:0 }\end{array}$ & nd & nd & 0,17 \\
\hline $\begin{array}{l}\text { Ác. Palmítico } \\
\text { C 16:0 }\end{array}$ & 20,18 & 23,60 & 19,60 \\
\hline $\begin{array}{l}\text { Ác. Esteárico } \\
\text { C 18:0 }\end{array}$ & 20,61 & 13,37 & 17,20 \\
\hline $\begin{array}{l}\text { Ác. Oleico } \\
\text { C 18:1 }\end{array}$ & 41,09 & 45,60 & 41,20 \\
\hline $\begin{array}{l}\text { Ac. Linolêico } \\
\text { C 18:2 }\end{array}$ & 14,08 & 8,00 & 18,60 \\
\hline $\begin{array}{l}\text { Ac. Araquidônico } \\
\text { C 20:4n6 }\end{array}$ & nd & nd & 0,76 \\
\hline $\begin{array}{l}\text { Ac. Araquídico } \\
\text { C 20:0 }\end{array}$ & 1,57 & nd & - \\
\hline
\end{tabular}

\section{Estimativa da composição triacilglicerídica}

Os valores da composição dos triacilglicerídeos calculados via software quando comparados com os obtidos por HT-HRGC/FID (Tabela 2) apresentam boa concordância, tendo em vista que o software emprega apenas a composição dos ácidos graxos para os cálculos.

Tabela 2. Composição de triacilglicerídeos do óleo de Nim (A. indica) obtido por dois métodos

\begin{tabular}{lcc}
\hline Triacilglicerídeos & $\begin{array}{c}\text { HT-HRGC/FID } \\
(\%)\end{array}$ & $\begin{array}{c}\text { Calculado por software } \\
(\%)\end{array}$ \\
\hline T48 & 0,29 & 0,82 \\
T50 & 7,70 & 9,26 \\
T52 & 34,70 & 34,98 \\
T54 & 45,30 & 44,98 \\
T56 & 4,40 & 2,72 \\
\hline
\end{tabular}

\section{CONCLUSÕES}

A HT-HRGC-FID mostrou-se uma técnica adequada, rápida e de baixo custo, pois não é necessário o emprego de etapas de derivatização para determinar a composição de TAG do óleo de Nim (A. indica).

As diferenças apresentadas com os dados de literatura devem-se ao fato que a composição do óleo de Nim (A. indica) é variável em função da variedade da planta, do solo, clima e tratos culturais.

O óleo de Nim (A. indica), que apresenta características muito interessantes, pode ser fácil e rapidamente hidrolisado, empregandose água subcrítica.

Pelos dados obtidos (Tabela 2) os triacilglicerídeos são dados majoritariamente por T54 e T52 ( 80\%) e entre os ácidos graxos, o ácido oleico $(\sim 41 \%)$ dado na Tabela 1.

O software empregado mostrou-se adequado ao cálculo da composição triacilglicerídica, pois houve concordância dos resultados (Tabela 2).

As vantagens deste método alternativo frente ao método tradicional ${ }^{13}$ são de não utilizar solventes orgânicos e catalisadores ácidos ou básicos, bem como não ser necessário efetuar as várias etapas de preparação da amostra para análise. Sendo assim, este método está de acordo com os conceitos da Química Analítica Verde.

\section{AGRADECIMENTOS}

Os autores agradecem o Prof. Dr. J. D. Fontana (UFPR) pelo padrão de trioleína e à CAPES, pela bolsa concedida.

\section{REFERÊNCIAS}

1. Siddiqui, B. S.; Afshan, F.; Ghiasuddin-Faizi, S.; Naqvi, S. N. H.; Tariq, R. M.; Phytochemistry 2000, 53, 371.

2. dos Santos, T. M.; Costa, N. P.; Torres, A. L.; Junior, A. L. B.; Pesq. Agropec. Bras. 2004, 39, 1071.

3. Sarais, G.; Angioni, A.; Lai, F.; Cabras, P.; Caboni, P.; J. Agric. Food Chem. 2009, 57, 2457.

4. Priyadarsini, R. V.; Manikandan, P.; Kumar, G. H.; Nagini, S.; Free Radical Res. 2009, 43, 492.

5. Dhar, R.; Zhang, K.; Talwar, G. P.; Garg, S.; Kumar, N.; J. Ethnopharmacol. 1998, 61, 31 .

6. Gurdeniz, G.; Ozen, B.; Tokatli, F.; Eur. Food Res. Technol. 2008, 227, 1275.

7. Marekov, I.; Tarandjiiska, R.; Momchilova, S.; Nikolova-Damyanova, B.; J. Liquid Chromatogr. Relat. Technol. 2008, 31, 1959.

8. Pereira A. D. S.; Bicalho, B.; Neto F. R. D. A.; Apidologie 2003, 174, 85. 
9. Pereira, A. D. S.; Padilha, M. C.; Neto, F. R. D. A.; Microchem. J. 2004, 77, 141.

10. Pinto, J. S. S.; Lanças, F. M.; J. Braz. Chem. Soc. 2006, 17, 85.

11. Moquin, P. H. L.; Temelli, F.; J. Supercrit. Fluids 2008, 45, 94.

12. Ackelsberg, O. J.; J. Am. Oil Chem. Soc. 1958, 35, 635.

13. Hartman, L.; Lago, R. C. A.; Lab. Pract. 1973, 22, 475.

14. Holliday, R. L.; King, J. W.; List, G. R.; Ind. Eng. Chem. Res. 1997, 36, 932.

15. Yu, Y.; Lou, X.; Wu, H.; Energy Fuels 2008, 22, 46.

16. Wiboonsirikul, J.; Hata, S.; Tsuno, T.; Kimura, Y.; Adachi, S.; LWT-Food Sci. Technol. 2007, 40, 1732.

17. Wiboonsirikul, J.; Kimura, Y.; Kanaya, Y.; Tsuno, T.; Adachi, S.; Biosci. Biotechnol. Biochem. 2008, 72, 384.

18. Sereewatthanawut, I.; Prapintip, S.; Watchiraruji, K.; Goto, M.; Sasaki, M.; Shotipruk, A.; Bioresour. Technol. 2008, 99, 555.
19. Kumar, S.; Gupta, R. B.; Ind. Eng. Chem. Res. 2009, 47, 9321.

20. Rogalinski, T.; Liu, K.; Albrecht, T.; Brunner, G.; J. Supercrit. Fluids 2008, 46, 335 .

21. Rogalinski, T.; Herrmann, S.; Brunner, G.; J. Supercrit. Fluids 2005, 36, 49.

22. Moreschi, S. R. M.; Petenate, A. J.; Meireles, M. A. A.; J. Agric. Food Chem. 2004, 52, 1753.

23. Koel, M.; Kaljurand, M.; Pure Appl. Chem. 2006, 78, 1993.

24. Armenta, S.; Garrigues, S.; de la Guardia, M.; Trends Anal. Chem. 2008, 27, 497.

25. Antoniosi, N. R.; Tese de Doutorado, Universidade de São Paulo, Brasil, 1995.

26. Antoniosi, N. R.; Mendes, O. L.; Lanças, F. M.; Chromatographia 1995, $40,557$. 